\title{
EDITORIAL
}

\section{From clot to collagen: coagulation peptides in interstitial lung disease}

\author{
K. Dabbagh, R.C. Chambers, G.J. Laurent
}

The fragile architecture of the lung is constantly threatened by a barrage of insults originating from both air and blood interfaces. The necessity to protect our lungs has lead to the evolution of exquisite defence systems. These comprise a complex screen of antioxidants, a mucocilliary escalator which is constantly clearing the respiratory tract and sophisticated immune defence mechanisms. When the insult involves vascular damage, the coagulation cascade is activated and is responsible for preventing blood loss at sites of injury by promoting the generation of a fibrin clot. This is vital for haemostasis, but chronic activation of this cascade with persistent fibrin deposition is frequently observed in respiratory diseases involving both the airways (e.g. asthma, chronic obstructive pulmonary disease) and the lung parenchyma (e.g. interstitial lung disease). It is often speculated that these events might be important in the pathogenesis of these diseases, but solid evidence for this has been scarce and, until quite recently, researchers have failed to provide data which could link the features of these diseases (i.e. chronic inflammation and tissue repair) to any known actions of coagulation cascade proteins.

In this article, we will review research developments which suggest an ever increasing range of cellular functions for elements of the coagulation cascade. These functions are such that many of the pro-inflammatory and fibroproliferative events which are commonly observed in respiratory diseases, and are most commonly ascribed to elements of a cytokine network, could in fact be orchestrated by proteins of the coagulation cascade. This article will focus on the relevance of these proteins in interstitial lung diseases in which tissue repair and fibrosis are commonly observed.

\section{The coagulation cascade}

The coagulation cascade is usually only initiated upon damage to the endothelium. The main processes of this cascade have been reviewed recently [1], but are briefly discussed below and summarized in figure 1. The coagulation cascade comprises two pathways termed the extrinsic and the intrinsic pathways. The extrinsic pathway is triggered by endothelial cell expression of tissue factor

Centre for Cardiopulmonary Biochemistry and Respiratory Medicine, UCL Medical School, Rayne Institute, London, UK.

Correspondence: R.C. Chambers, Centre for Cardiopulmonary and Respiratory Medicine, UCL Medical School, Rayne Institute, 5 University Street, London WC1E 6JJ, UK. Fax: 441712096973.

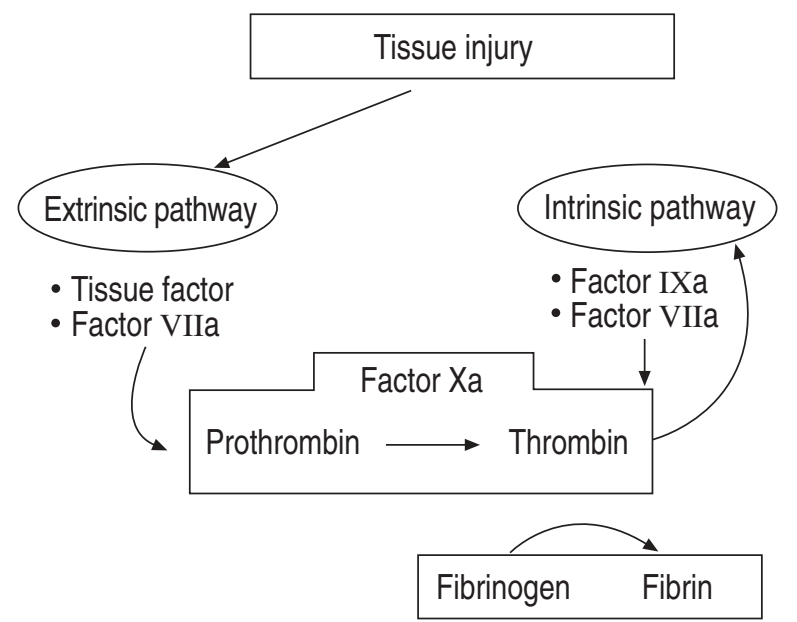

Fig. 1. - From tissue injury to fibrin deposition: key steps in the coagulation cascade.

(TF) at sites of vascular injury. TF acts as a receptor for factor VII which is converted to active VIIa. This complex then activates factor $\mathrm{X}$ which, together with factor Va, is one of the main components of the prothrombinase complex, res-ponsible for converting prothrombin to active thrombin. Thrombin in turn converts soluble fibrinogen into insoluble fibrin. The extrinsic pathway serves to initiate the co-agulation cascade, but it is the intrinsic pathway that is responsible for sustained blood coagulation. This pathway is maintained by the action of factor $\mathrm{Xa}$ and thrombin which are responsible for the activation of factors XII, XI and IX. These in turn lead to prolonged generation of the components of the prothrombinase complex and sustained generation of fibrin. The intrinsic pathway is also responsible for the formation of active factor XIII, which in turn stabilizes and cross-links the nascent fibrin clot.

The amount of fibrin present at sites of tissue injury is tightly regulated by the actions of initiators and inhibitors of the coagulation cascade, as well as the fibrinolytic pathway, responsible for the degradation of the clot, but which will not be discussed further here. Inhibitors include proteins such as lipoprotein-associated coagulation inhibitor and extrinsic pathway inhibitor, responsible for the early shut-down of the extrinsic pathway; whereas antithrombin III is the major inhibitor of the intrinsic pathway due to its ability to block the activities of thrombin and factors IXa, $\mathrm{Xa}$ and XIa. Finally, activated protein $\mathrm{C}$ acts as another 
major inhibitor of the intrinsic pathway by blocking the prothrombinase complex and further thrombin activation.

\section{Involvement of coagulation cascade proteins in interstitial lung disease}

The main function of the coagulation cascade is to limit blood loss and to provide a temporary matrix which will form the structural basis for subsequent repair processes. These processes include the migration and proliferation of inflammatory and mesenchymal cells, followed by the synthesis of extracellular matrix proteins. There is some evidence that persistent activation of the coagulation cascade may play a role in sustained inflammation in the lungs of patients with interstitial lung disease. For example, extensive alveolar fibrin deposition and hyaline membrane formation, as well as inflammatory cell infiltration, are commonly observed in patients with adult respiratory distress syndrome (ARDS) or infantile respiratory distress syndrome [2]. A significant proportion of these patients develop fibrotic lesions in the terminal stages of the disease, characterized by increased mesenchymal cell proliferation and collagen deposition. Supporting, but not providing direct evidence for a role for coagulation cascade proteins in these diseases, several studies have shown that there is also increased procoagulant activity in the lungs and bronchoalveolar lavage fluid from patients with these conditions $[3,4]$. Similar findings have been reported for patients with chronic interstitial lung disease [5], including patients with idiopathic pulmonary fibrosis (IPF), as well as with pulmonary fibrosis associated with systemic sclerosis (SSc) [6, 7]. Consistent with these findings, we and others have shown that thrombin levels are increased in bronchoalveolar lavage (BAL) fluid obtained from patients with pulmonary fibrosis associated with SSc $[8,9]$, and that thrombin is a major fibroblast mitogen present in these fluids [9]. Similar findings have also been reported for animal models of fibrosis. For example, thrombin has been shown to be increased and act as a potent fibroblast mitogen in BAL fluid obtained from rats developing fibrosis following bleomycin administration [10]. More recently, TF expression was shown to be increased at sites characterized by persistent fibrin deposition and fibroproliferation in mice following a similar administration of bleomycin [11].

These studies suggest the involvement of coagulation proteins in these conditions but evidence that they may be playing a direct role in the pathogenesis of these diseases has been obtained in studies in which these products or their inhibitors were administered to experimental animals. For example, intravenous administration of fibrin(ogen) degradation products has been shown to lead to increases in endothelial permeability, extravasation of blood proteins, as well as interstitial and alveolar clot formation [12]. Similar lung changes have also been observed following intravenous infusion of thrombin in sheep [13]; whilst thrombin inhibitors have been shown to be efficacious at reducing lung procoagulant activity and alveolar fibrin deposition in animal models of endotoxaemia in pigs [14], as well as reducing the severity of acute lung injury in newborn piglets [15], although the final outcome of the lesion obtained in this particular model was not reported. Taken together, these studies suggest an impor- tant role for these proteins in the pathophysiology of these conditions. The crucial question today, therefore, seems to be how are these proteins causing pulmonary inflammation and disease?

\section{Role of coagulation cascade proteins in pulmonary inflammation and repair}

Earlier studies proposed roles for fibrinogen, fibrin and fibrin(ogen) degradation products in chronic disease as a result of their effects on the pulmonary endothelium and the recruitment of inflammatory cells. Indeed, low molecular weight degradation products of fibrinogen, which are generated during the formation of fibrin clots by thrombin, have been demonstrated to increase vascular permeability [16] and to stimulate neutrophil chemotaxis [17]. In addition, thrombin itself can exert similar effects [18, 19]. Furthermore, the severity of pulmonary inflammation can be reduced by neutrophil depletion in animals infused with fibrinogen degradation products [20] and thrombin [21] supporting a direct role for coagulation proteins in the recruitment of these cells in lung inflammation. Thrombin is also a potent chemoattractant for macrophages [22], which accumulate in the lungs and BAL fluids of patients with interstitial lung disease [23] and are believed to play an important role in these conditions by producing proinflammatory cytokines and growth factors. More recently, CIRINo et al. [24] also provided convincing evidence that factor Xa has potent pro-inflammatory effects in vivo by demonstrating that subplantar injection of factor Xa results in an inflammatory reaction characterized by oedema formation and mast cell infiltration. Although this study was not carried out in the lung, factor Xa may exert similar effects there, and may be particularly important in conditions associated with mast cell recruitment and activation.

Coagulation proteins are also known to affect mesenchymal cells and tissue repair processes (for a summary of these cellular effects see figure 2). Thrombin is a potent chemoattractant and mitogen, both for fibroblasts [25] and smooth muscle cells [26] and stimulates them to produce cytokines and growth factors, including platelet-derived growth factor (PDGF) [8], interleukin (IL)-6 [27], basic fibroblast growth factor (bFGF) [28] and transforming growth factor- $\beta_{1}$ (TGF- $\beta_{1}$ ) [29]. Furthermore, we have

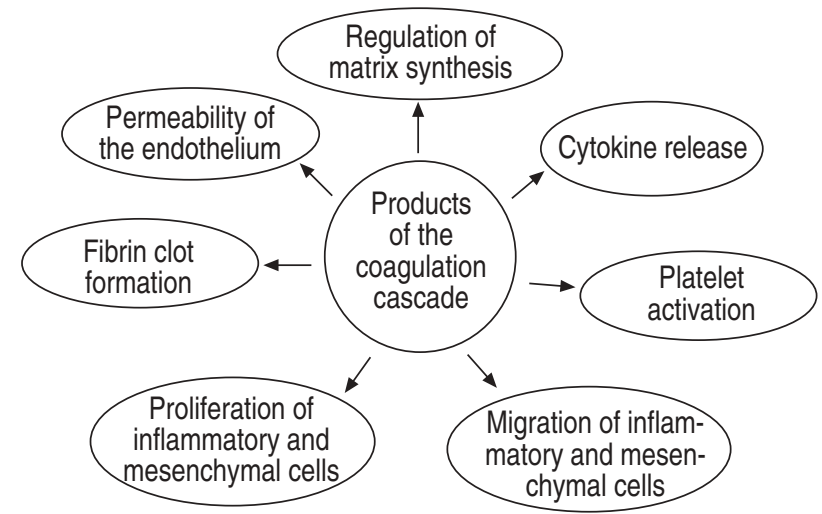

Fig. 2. - Pluripotent biological effects of products of the coagulation cascade. 
recently shown that thrombin is a potent stimulator of smooth muscle cell [30] and fibroblast (Chambers et al., unpublished data) procollagen production and gene expression. These cellular effects of thrombin may play an important role in sustaining inflammation at sites of lung injury and may also be important in the development of fibrotic lesions, which are commonly observed in patients with interstitial lung disease.

Factor Xa, fibrin, and fibrin(ogen) degradation products have similarly been shown to exert a number of cellular effects. For example, factor $\mathrm{Xa}$ is a smooth muscle cell mitogen [31] and stimulates growth factor production [32]. Fibrinogen degradation products stimulate fibroblast proliferation [33], whereas fibrin stimulates macrophage to secrete IL-1 $\beta$ [34]. Conversely, inflammatory cytokines, such as IL-6 have been shown to induce the secretion of fibrinogen by alveolar epithelial cells [35]. In addition, type II epithelial cells have been shown to be major sources of TF. Cells expressing tissue factor have been located close to areas associated with fibrin deposition and fibroblastic foci in the lungs of patients with pulmonary fibrosis. These findings raise the possibility that locally produced tissue factor can cause activation of the extrinsic coagulation cascade and sustained alveolar clot formation in the absence of vascular injury. Taken together these data strongly suggest that these coagulation proteins can play direct roles at all stages of the development of interstitial lung disease, from the initial vascular leakage to the development of the final fibrotic lesion.

\section{Conclusions and future directions}

It is now clear that products of the coagulation cascade exert a wide range of biological activities. In this article, we propose that higher organisms have developed an integrated network in which coagulation cascade proteins, as well as cytokines, regulate the key process of inflammation and repair. However, the precise relationship between these different polypeptide mediators continues to be investigated, and it is likely that coagulation proteins are present at the very earliest stages of disease and may perpetuate inflammation and repair processes by inducing the recruitment, as well as, the proliferation of inflammatory and mesenchymal cells in chronic lung disease. In addition, they can also induce the release of pro-inflammatory and profibrotic cytokines, which in turn contribute to sustaining inflammation and repair processes. Coagulation cascade proteins and their receptors may, therefore, represent potential targets for therapeutic intervention in interstitial lung diseases. Current research efforts are focused on developing agents that are capable of blocking the cellular effects of these proteins with minimal haemostatic complications. Their usefulness as therapeutic agents awaits confirmation, but this information should be forthcoming as we continue to unravel the function of coagulation proteins in disease processes and the receptor systems through which they act.

\section{References}

1. Davie EW. Biochemical and molecular aspects of the coagulation cascade. Thromb Haemost 1995; 74: 1-6.
2. Hasegawa N, Husari AW, Hart WT, Kandra TG, Raffin TA. Role of the coagulation system in ARDS. Chest 1994; 105: 268-277.

3. Idell S, Gonzalez K, Bradford H, et al. Procoagulant activity in bronchoalveolar lavage in the adult respiratory distress syndrome. Contribution of tissue factor associated with factor VII. Am Rev Respir Dis 1987; 136; 1466-1474.

4. Chapman HA, Stahl M, Allen CL, Yee R, Fair DS. Regulation of the procoagulant activity within the bronchoalveolar compartment of normal human lung. Am Rev Respir Dis 1988; 137: 1417-1425.

5. Chapman HA, Allen CL, Stone OL. Abnormalities in pathways of alveolar fibrin turnover among patients with interstitial lung disease. Am Rev Respir Dis 1986; 133: 437-443.

6. Kotani I, Sato A, Hayakawa H, Urano T, Takada Y, Takada A. Increased procoagulant and antifibrinolytic activities in the lungs with idiopathic pulmonary fibrosis. Thromb Res 1995; 77: 493-504.

7. Imokawa S, Sato A, Hayakawa H, Kotani I, Urano T, Takada A. Tissue factor expression and fibrin deposition in the lungs of patients with idiopathic pulmonary fibrosis and systemic sclerosis. Am J Respir Crit Care Med 1997; 156: 631-636.

8. Ohba T, McDonald JK, Silver RM, Strange C, LeRoy EC, Ludwicka A. Scleroderma bronchoalveolar lavage fluid contains thrombin, a mediator of human lung fibroblast proliferation via induction of platelet-derived growth factor alpha-receptor. Am J Respir Cell Mol Biol 1994; 10: 405-412.

9. Hernandez-Rodriguez NA, Cambrey AD, Harrison NK, et al. Role of thrombin in pulmonary fibrosis. Lancet 1995; 346: 1071-1073.

10. Tani K, Yasuoka S, Ogushi F, et al. Thrombin enhances lung fibroblast proliferation in bleomycin-induced pulmonary fibrosis. Am J Respir Cell Mol Biol 1991; 37: 3948.

11. Olman MA, Mackman N, Gladson CL, Moser KM, Loskutoff DJ. Changes in procoagulant and fibrinolytic gene expression during bleomycin-induced lung injury in the mouse. J Clin Invest 1995; 96: 1621-1630.

12. Manwaring D, Thorning D, Curreri PW. Mechanisms of acute pulmonary dysfunction induced by fibrinogen degradation product D. Surgery 1978; 84: 45-54.

13. Lo SK, Perlman MB, Niehaus GD, Malik AB. Thrombininduced alterations in lung fluid balance in awake sheep. J Appl Physiol 1985; 58: 1421-1427.

14. Hoffmann H, Siebeck M, Spannagl M, et al. Effect of recombinant hirudin, a specific inhibitor of thrombin, on endotoxin-induced intravascular coagulation and acute lung injury in pigs. Am Rev Respir Dis 1990; 142: 782788.

15. Schmidt B, Davis P, LA Pointe H, Monkman S, Coates G, $\mathrm{deSa}$ D. Thrombin inhibitors reduce intrapulmonary accumulation of fibrinogen and procoagulant activity of bronchoalveolar lavage fluid during acute lung injury induced by pulmonary overdistention in newborn piglets. Pediatr Res 1996; 39: 798-804.

16. Sueishi K, Nanno S, Tanaka K. Permeability enhancing and chemotactic activities of lower molecular weight degradation products of human fibrinogen. Thromb Haemost 1981; 45: 90-94.

17. Senior RM, Skogen WF, Griffin GL, Wilner GD. Effects of fibrinogen derivatives upon the inflammatory response. Studies with human fibrinopeptide B. J Clin Invest 1986; 77: 1014-1019. 
18. Bizios R, Lai L, Fenton JW II, Malik AB. Thrombininduced chemotaxis and aggregation of neutrophils. $J$ Cell Physiol 1986; 128: 485-490.

19. DeMichele MA, Minnear FL. Modulation of vascular endothelial permeability by thrombin. Semin Thromb Hemost 1992; 18: 287-295.

20. Manwaring D, Curreri PW. Cellular mediation of respiratory distress syndrome induced by fragment D. Ann Chir Gynaecol 1981; 70: 304-307.

21. Tahamont MV, Malik AB. Granulocytes mediate the increase in pulmonary vascular permeability after thrombin embolism. J Appl Physiol 1983; 54: 1489-1495.

22. Bar-Shavit R, Kahn A, Fenton JW II, Wilner GD. Receptor-mediated chemotactic response of a macrophage cell line (J774) to thrombin. Lab Invest 1983; 49: 702-707.

23. Weinberger SE, Kelman JA, Elson NA, et al. Bronchoalveolar lavage in interstitial lung disease. Ann Intern Med 1978; 89: 459-466.

24. Cirino G, Cicala C, Bucci M, et al. Factor Xa as an interface between coagulation and inflammation. Molecular mimicry of factor Xa association with effector cell protease receptor-1 induces acute inflammation in vivo. $J$ Clin Invest 1997; 99: 2446-2451.

25. Chen LB, Buchanan JM. Mitogenic activity of blood components. I. Thrombin and prothrombin. Proc Natl Acad Sci USA 1975; 72: 131-135.

26. Bar-Shavit R, Benezra M, Eldor A, et al. Thrombin immobilized to extracellular matrix is a potent mitogen for vascular smooth muscle cells: nonenzymatic mode of action. Cell Regul 1990; 1: 453-463.

27. Sower LE, Froelich CJ, Carney DH, Fenton JW II, Klimpel GR. Thrombin induces IL-6 production in fibroblasts and epithelial cells. Evidence for the involvement of the seven-transmembrane domain (STD) receptor for alphathrombin. J Immunol 1995; 155: 895-901.
28. Samaniego F, Markham PD, Gendelman R, Gallo RC, Ensoli B. Inflammatory cytokines induce endothelial cells to produce and release basic fibroblast growth factor and to promote Kaposi's sarcoma- like lesions in nude mice. $J$ Immunol 1997; 158: 1887-1894.

29. Bachhuber BG, Sarembock JJ, Gimple LW, Owens GK. Alpha-thrombin induces transforming growth factor- $\beta_{1}$ mRNA and protein in cultured vascular smooth muscle cells via a proteolytically activated receptor. $J$ Vasc Res 1997; 34: 41-48.

30. Dabbagh K, Laurent GJ, McAnulty RJ, Chambers RC. Thrombin stimulates smooth muscle cell procollagen synthesis and mRNA levels via a PAR-1 mediated mechanisms. Thromb Haemost 1998; 79: 405-409.

31. Gasic GP, Arenas CP, Gasic TB, Gasic GJ. Coagulation factors $\mathrm{X}, \mathrm{Xa}$, and protein $\mathrm{S}$ as potent mitogens of cultured aortic smooth muscle cells. Proc Natl Acad Sci USA 1992; 89: 2317-2320.

32. Ko FN, Yang YC, Huang SC, Ou JT. Coagulation factor $\mathrm{Xa}$ stimulates platelet-derived growth factor release and mitogenesis in cultured vascular smooth muscle cells of rat. J Clin Invest 1996; 98: 1493-1501.

33. Gray AJ, Bishop JE, Lever ME, Laurent GJ. A putative role for fibrin(ogen) cleavage products and thrombin as mediators of vascular remodelling. Eur Respir Rev 1993; 3: 650-654.

34. Perez RL, Roman J. Fibrin enhances the expression of IL-1 $\beta$ by human peripheral blood mononuclear cells. Implications in pulmonary inflammation. J Immunol 1995; 154: 1879-1887.

35. Guadiz G, Sporn LA, Goss RA, Lawrence SO, Marder VJ, Simpson-Haidaris PJ. Polarized secretion of fibrinogen by lung epithelial cells. Am J Respir Cell Mol Biol 1997; 17: 60-69. 